\title{
On the Barban-Davenport-Halberstam theorem: IX
}

\author{
by \\ C. Hooley (Cardiff)
}

We revisit the topic of the third article of this series* III, in which a generalized theorem of Barban-Davenport-Halberstam type was established for a wide class of sequences of essentially positive density. Having on an intermediate occasion [4] derived an improved proof, we now consider the possibility of an asymptotic formula that stands in the same relationship to the result in III as does the Barban-Montgomery theorem described in I to the Barban-Davenport-Halberstam theorem itself. Commencing as before by letting $s$ denote, generally, a member of a given (strictly) increasing sequence of positive integers and letting

$$
S(x ; a, k)=\sum_{\substack{s \leq x \\ s \equiv a, \bmod k}} 1
$$

for any positive $k$ and any non-negative integer $a$, we still assume Criterion $\mathrm{U}$ to the effect that, for any positive integer A, the sequence s has the property that

$$
S(x ; a, k)=g\{k,(a, k)\} x+O\left(x \log ^{-A} x\right)
$$

as $x \rightarrow \infty$, where $(a, k)$ is the highest common factor of $a$ and $k$ and the constant implied by the $O$-notation depends at most on $A$. Then, still setting

$$
E(x ; a, k)=S(x ; a, k)-g\{k,(a, k)\} x
$$

and

$$
G(x, Q)=\sum_{k \leq Q} \sum_{0<a \leq k} E^{2}(x ; a, k) \quad(Q \leq x),
$$

we go beyond the previously obtained bound

$$
G(x, Q)=O(Q x)+O\left(x^{2} \log ^{-A} x\right)
$$

by demonstrating the asymptotic formulae

* We refer to these articles by the Roman numeral indicating their position in the series; their full particulars are given in the list of references at the end. 


$$
\begin{aligned}
G(x, Q)=\left(D_{1}+o(1)\right) Q x+O\left(x^{2} \log ^{-A} x\right) \\
\quad(Q \leq x ; o(1) \rightarrow 0 \text { as } x / Q \rightarrow \infty)
\end{aligned}
$$

and

$$
G(x, Q)=D_{2} Q x+O\left(x^{2} \log ^{-A} x\right) \quad(Q=x)
$$

when the sequences satisfy the further Criterion $\mathrm{S}$ that states that the function $g(k)=g(k, k)$ in the statement of Criterion $\mathrm{U}$ is of the form $C \psi(k)$, where $C>0$ and $\psi(k)$ is a multiplicative function. Thus the sequences previously considered are subject to a generalized Barban-Montgomery theorem if the events $p \mid s$ and $q \mid s$ for distinct primes $p$ and $q$ be inherently statistically independent. Stemming from these criteria, one relation needed later is derived at once because it is the basis of an interesting property of the sequence that we should not allow to pass unnoticed. This arises from a comparison, for any divisor $\delta$ of $k$, of the equation

$$
\sum_{\substack{s \leq x \\(s, \bar{k})=\delta}} 1=\phi(k / \delta) g(k, \delta) x+O\left(k x \log ^{-A} x\right)
$$

derived from Criterion $\mathrm{U}$ with the representation of its left-hand side as

$$
\begin{aligned}
\sum_{\substack{s \leq x \\
s \equiv 0, \bmod \delta}} \sum_{(s / \delta, k / \delta)=1} 1 & =\sum_{\substack{s \leq x \\
s \equiv 0, \bmod \delta}} \sum_{d \delta|s, d| k / \delta} \mu(d) \\
& =\sum_{d \mid k / \delta} \mu(d) \sum_{\substack{s \leq x \\
s \equiv 0, \bmod d \delta}} 1 \\
& =x \sum_{d \mid k / \delta} \mu(d) g(d \delta)+O\left(d(k / \delta) x \log ^{-A} x\right) .
\end{aligned}
$$

On equating these two expressions and letting $x \rightarrow \infty$ after a division by $x$, we conclude that

$$
\phi(k / \delta) g(k, \delta)=\sum_{d \mid k / \delta} \mu(d) g(d \delta)
$$

and first recoup the result of Lemma 15 in [4] by substituting this in (5). But, if Criterion $\mathrm{S}$ be also assumed, the impact of the multiplicativity of $g(k) / C$ on the above equation is that $g(k, \delta) / C$ is also multiplicative in the sense that the sequence has the property that, as $x \rightarrow \infty$,

$$
S(x ; a, k)=C \psi(k, a) x+O\left(x \log ^{-A} x\right),
$$

where $\psi(k, a)$ is a multiplicative function of $k$ for each integer a. Equivalently, this may be expressed as being the requirement that Criterion $\mathrm{S}$ be satisfied not only by the sequence of numbers $s$ but also by each sequence of numbers $s+b$ for each positive integer $b$. Yet in the present work we prefer 
not to avail ourselves of this property, which in itself is weaker than Criteria $\mathrm{U}$ and $\mathrm{S}$ taken together.

Our treatment of the Barban-Montgomery theorem in I combined an appeal to the Barban-Davenport-Halberstam theorem with a technique that partially described the behaviour of

$$
\sum_{k \leq Q} \sum_{\substack{0<a \leq k \\(a, k)=1}} \theta^{2}(x ; a, k)
$$

for large $Q$ by means of the prime-number theorem for arithmetical progressions with small moduli $l \leq x / Q$. Here we adopt an initially similar framework based on the generalized Theorem 1 of III together with an analysis of $G(x, Q)$ that depends on Criterion $\mathrm{U}$ for small moduli, even though our path must then diverge from that taken in I when we come to calculate the sums that arrive after the initial analysis. The principal difficulty in regard to these sums being the generality of the situation in which they are estimated, we exploit, inter alia, the positive density $C$ of the given sequence by means of two simple arguments, the second of which depends on a sieve method. Also, not unexpectedly, the final use of contour integration involves complications not present in its counterpart in I.

Throughout the letter $A$ denotes a positive absolute constant that is not necessarily the same at each occurrence and on which the constants implied by the $O$-notation at most depend save in one explicit instance. The letter $s$ not only denotes a member of the given sequence but also a complex variable $\sigma+i t$, it being clear from the context which meaning is intended; the function $\sigma_{-1}(l)$ is, as usual, the sum of the reciprocals of the divisors of $l$.

It is enough to treat the case $Q>x \log ^{-A} x$ for any value of $A$ chosen in the statement of (2) and (3) because in the opposite case the proposed result (2) is contained in the generalized Barban-Davenport-Halberstam inequality (1) proved in III and [4]. Having thus isolated the situation needing serious attention, we set

$$
G\left(x ; Q_{1}, Q_{2}\right)=G\left(x, Q_{2}\right)-G\left(x, Q_{1}\right)=\sum_{Q_{1}<k \leq Q_{2}} \sum_{0<a \leq k} E^{2}(x ; a, k)
$$

for

$$
Q_{1}=x \log ^{-A} x<Q_{2} \leq x
$$

so that

$$
G\left(x, Q_{2}\right)=G\left(x ; Q_{1}, Q_{2}\right)+O\left(x^{2} \log ^{-A} x\right)
$$

by a further application of (1), wherein the substitution of $Q_{2}$ for $Q$ is a 
notational convenience. Next, by equation (163) in [4] in which

$$
H(x, k)=\sum_{0<a \leq k} E^{2}(x ; a, k),
$$

we have

$$
\begin{aligned}
H(x, k) & =\sum_{0<a \leq k} S^{2}(x ; a, k)-x^{2} \sum_{\delta \mid k} \phi(k / \delta) g^{2}(k, \delta)+O\left(\frac{x^{2} d_{3}(k)}{k \log ^{A+4} x}\right) \\
& =\sum_{0<a \leq k} S^{2}(x ; a, k)-x^{2} \frac{P(k)}{k}+O\left(\frac{x^{2} d_{3}(k)}{k \log ^{A+4} x}\right), \quad \text { say }
\end{aligned}
$$

wherefore, having replaced the notation $P(k) / k$ by $C^{2} M(k)$ and set

$$
T(u)=\sum_{k \leq u} M(k),
$$

we infer that

$$
\begin{aligned}
G\left(x ; Q_{1}, Q_{2}\right)= & \sum_{Q_{1}<k \leq Q_{2}} \sum_{0<a \leq k} S^{2}(x ; a, k)-C^{2} x^{2}\left\{T\left(Q_{2}\right)-T\left(Q_{1}\right)\right\} \\
& +O\left(x^{2} \log ^{-A} x\right) \\
= & \Gamma\left(x ; Q_{1}, Q_{2}\right)-C^{2} x^{2}\left\{T\left(Q_{2}\right)-T\left(Q_{1}\right)\right\} \\
& +O\left(x^{2} \log ^{-A} x\right), \text { say. }
\end{aligned}
$$

We transform in turn the sum $\Gamma\left(x ; Q_{1}, Q_{2}\right)$, whose inner sum equals

$$
\begin{aligned}
\sum_{0<a \leq k} \sum_{\substack{s, s^{\prime} \leq x \\
s \equiv s^{\prime} \equiv a, \bmod k}}=\sum_{\substack{s, s^{\prime} \leq x \\
s \equiv s^{\prime}, \bmod k}} 1=\sum_{s \leq x} 1+2 \sum_{\substack{s^{\prime}<s \leq x \\
s \equiv s^{\prime}, \bmod k}} 1 \\
=C x+2 \sum_{\substack{s^{\prime}<s \leq x \\
s \equiv s^{\prime}, \bmod k}} 1+O\left(x \log ^{-A} x\right)
\end{aligned}
$$

by Criteria $\mathrm{U}$ and $\mathrm{S}$ for the special case $k=1$. Thus, by (7),

$$
\begin{aligned}
\Gamma\left(x ; Q_{1}, Q_{2}\right)= & \left(Q_{2}-Q_{1}+O(1)\right) C x+2 \sum_{Q_{1}<k \leq Q_{2}} \sum_{\substack{s^{\prime}<s \leq x \\
s \equiv s^{\prime}, \bmod k}} 1 \\
& +O\left(x^{2} \log ^{-A} x\right) \\
= & C Q_{2} x+2 J\left(x ; Q_{1}, Q_{2}\right)+O\left(x^{2} \log ^{-A} x\right), \quad \text { say },
\end{aligned}
$$

with which equation the preliminary analysis of our problem is concluded.

To manage the second term on the final line of (12) we consider the sum $J(x, Q)=J(x ; Q, x)$ for

$$
x \log ^{-A} x<Q \leq x
$$


through which $J\left(x ; Q_{1}, Q_{2}\right)$ is expressed by the equation

$$
J\left(x ; Q_{1}, Q_{2}\right)=J\left(x, Q_{1}\right)-J\left(x, Q_{2}\right) .
$$

The conditions of summation in this double sum imply that $s-s^{\prime}=l k$ for some positive integer $l$, by means of which it is seen that the former conditions are tantamount to the combination of the latter condition with the requirement that $l<x / Q$ and $s^{\prime}+l Q<s \leq x$. Therefore

$$
\begin{aligned}
J(x, Q) & =\sum_{l<x / Q} \sum_{\substack{s^{\prime}+l Q<s \leq x \\
s \equiv s^{\prime}, \bmod l}} 1 \\
& =\sum_{l<x / Q} \sum_{\delta \mid l} \sum_{\substack{0<b \leq l \\
(b, l)=\delta}} \sum_{\substack{s^{\prime}<x-l Q \\
s^{\prime} \equiv b, \bmod l \\
l}} 1 \\
& =\sum_{\substack{s^{\prime}+l Q<s \leq x \\
s \equiv b, \bmod l}} \sum_{\delta \mid l} \sum_{\substack{0<b \leq l \\
(b, l)=\delta}} K(x, Q, b, l)=\sum_{0<l<x / Q} L(x, Q, l), \quad \text { say. }
\end{aligned}
$$

Here, since Criterion $U$ and the consequential inequality

$$
g(l, \delta)=O(1 / l)
$$

proved in III imply that

$$
S(y ; b, l)=g\{l,(b, l)\} y+O\left(x \log ^{-2 A} x\right)
$$

when $0<y \leq x$, we have

$$
\begin{aligned}
K(x, Q, b, l) & =g(l, \delta) \sum_{\substack{s^{\prime}<x-l Q \\
s^{\prime} \equiv b, \bmod l}}\left(x-l Q-s^{\prime}\right)+O\left(\frac{x}{\log ^{2 A} x} \sum_{\substack{s^{\prime} \leq x \\
s^{\prime} \equiv b, \bmod l}} 1\right) \\
& =g(l, \delta) \int_{0}^{x-l Q} S(t ; b, l) d t+O\left(\frac{x^{2}}{l \log ^{2 A} x}\right) \\
& =g(l, \delta)\left\{\frac{1}{2} g(l, \delta)(x-l Q)^{2}+O\left(\frac{x^{2}}{\log ^{2 A} x}\right)\right\}+O\left(\frac{x^{2}}{l \log ^{2 A} x}\right) \\
& =\frac{1}{2} g^{2}(l, \delta)(x-l Q)^{2}+O\left(\frac{x^{2}}{l \log ^{2 A} x}\right)
\end{aligned}
$$

by another application of (16). Consequently, by a summation over $b$ and $\delta$,

$$
\begin{aligned}
L(x, Q, l) & =\frac{1}{2}(x-l Q)^{2} \sum_{\delta \mid l} \phi(l / \delta) g^{2}(l, \delta)+O\left(x^{2} \log ^{-2 A} x\right) \\
& =\frac{1}{2} C^{2}(x-l Q)^{2} M(l)+O\left(x^{2} \log ^{-2 A} x\right)
\end{aligned}
$$


in the notation of (9), from which, (15), and (13) we obtain the equation

$$
\begin{aligned}
J(x, Q) & =\frac{1}{2} C^{2} \sum_{l<x / Q}(x-l Q)^{2} M(l)+O\left(x^{2} \log ^{-A} x\right) \\
& =\frac{1}{2} C^{2} x^{2} \sum_{l<x / Q}\left(1-\frac{l Q}{x}\right)^{2} M(l)+O\left(x^{2} \log ^{-A} x\right) \\
& =C^{2} x^{2} T^{*}(x / Q)+O\left(x^{2} \log ^{-A} x\right), \quad \text { say, }
\end{aligned}
$$

that contains a sum analogous to $T(u)$ in (10).

The time has come to bring Criterion $\mathrm{S}$ seriously into play by systematically expressing $g(k)$ as $C \psi(k)$, where $C>0$ and $\psi(k)$ is multiplicative. This is used to study the Dirichlet's series related to the sums $T(u)$ and $T^{*}(u)$, the appropriate properties of which are developed by considering the impact on $\psi(k)$ of two simple attributes of the sequence. Each property being related to the fact that our sequence is contained in the sequence of natural numbers, we restate a special case of (16) by remarking that

$$
S(x ; 0, k)=C \psi(k) x+O\left(x \log ^{-A} x\right) \leq[x / k]=x / k+O(1)
$$

for any given $k$ and that hence

$$
C \psi(k) \leq 1 / k
$$

by letting $x \rightarrow \infty$. Secondly, we consider the effect of eliminating from our sequence all multiples of primes $p$ belonging to a given finite set $\mathcal{S}$, to which end we let $d_{1}$ denote, generally, a square-free number (possibly 1) composed entirely of prime factors in $\mathcal{S}$. Then, for any (large) $x$, Legendre's exclusion principle yields

$$
\sum_{d_{1}} \mu\left(d_{1}\right) S\left(x ; 0, d_{1}\right) \leq \sum_{d_{1}} \mu\left(d_{1}\right)\left[\frac{x}{d_{1}}\right],
$$

from which by way of Criteria $\mathrm{U}$ and $\mathrm{S}$ we infer that

$$
C x \sum_{d_{1}} \mu\left(d_{1}\right) \psi\left(d_{1}\right)+O\left(x \log ^{-A} x\right) \leq x \sum_{d_{1}} \frac{\mu\left(d_{1}\right)}{d_{1}}+O(1)
$$

and then that

$$
C x \prod_{p \in \mathcal{S}}(1-\psi(p))+O\left(x \log ^{-A} x\right) \leq x \prod_{p \in \mathcal{S}}\left(1-\frac{1}{p}\right)+O(1)
$$

where here the constant implied by the $O$-notation depends at most on $A$ and $\mathcal{S}$. Hence, letting $x \rightarrow \infty$, we deduce the inequality

$$
\prod_{p \in \mathcal{S}}(1-\psi(p)) \leq \frac{1}{C} \prod_{p \in \mathcal{S}}\left(1-\frac{1}{p}\right)
$$


that is parallel to the inequality

$$
\prod_{p \in \mathcal{S}} p \psi(p) \leq \frac{1}{C}
$$

stemming from (18). To turn these relations into account let us set

$$
\psi(p)=\frac{1}{p}+\frac{\eta_{p}}{p}
$$

and denote by $\mathcal{S}_{1}, \mathcal{S}_{2}$, respectively, finite sets $\mathcal{S}$ for which $\eta_{p} \geq 0$ and $\eta_{p}<0$ throughout, using the symbols $\mathcal{S}_{1}^{\prime}, \mathcal{S}_{2}^{\prime}$ for the corresponding sets of all $p$ for which these inequalities hold. Then, by (20),

$$
\prod_{p \in \mathcal{S}_{1}}\left(1+\eta_{p}\right) \leq \frac{1}{C}
$$

so that

$$
\prod_{p \in \mathcal{S}_{1}^{\prime}}\left(1+\eta_{p}\right) \quad \text { and } \quad \prod_{p \in \mathcal{S}_{1}^{\prime}} \eta_{p}
$$

are convergent. Similarly, since

$$
\prod_{p \in \mathcal{S}_{2}}\left(1-\frac{\eta_{p}}{p-1}\right)=\prod_{p \in \mathcal{S}_{2}}\left(1+\frac{\left|\eta_{p}\right|}{p-1}\right) \leq \frac{1}{C}
$$

owing to (19), we see that

$$
\prod_{p \in \mathcal{S}_{2}^{\prime}}\left(1-\frac{\eta_{p}}{p-1}\right) \text { and } \sum_{p \in \mathcal{S}_{2}^{\prime}} \frac{\eta_{p}}{p-1}
$$

are (absolutely) convergent. Consequently,

$$
\prod_{p}\left(1+\frac{\eta_{p}^{2}}{p}\right) \text { and } \sum_{p} \frac{\eta_{p}^{2}}{p} \text { are absolutely convergent }
$$

since $\eta_{p}$ is certainly always $O(1)$; note here that a more symmetrical proof would be obtained by replacing the use of (20) by that of its corollary

$$
\prod_{p \in \mathcal{S}}(1+\psi(p)) \leq \frac{1}{C} \prod_{p \in \mathcal{S}}\left(1+\frac{1}{p}\right)
$$

that is parallel to (19), although the alternative procedure proposed would disguise the relative strength of (20) as compared with (22). Nothing more need now be extracted from this technique of comparison, albeit we shall see from some closing comments that its potentialities have been by no means exhausted. 
Next, equalling

$$
\sum_{\delta \mid l} \frac{1}{\phi(l / \delta)}\left(\sum_{d \mid l / \delta} \mu(d) \psi(d \delta)\right)^{2}
$$

in virtue of $(9),(6)$, and Criterion $\mathrm{S}$, the sum $M(l)$ is multiplicative and subject to the bound

$$
M(l)=O\left\{\frac{1}{l^{2}} \sum_{\delta \mid l} \phi\left(\frac{l}{\delta}\right)\right\}=O\left(\frac{1}{l}\right)
$$

that most easily follows directly from (9) and (16). Hence, for $\sigma>0$, the Dirichlet's series

$$
F(s)=\sum_{l=1}^{\infty} \frac{M(l)}{l^{s}}
$$

is absolutely convergent and is equal to

$$
\prod_{p}\left(1+\sum_{\alpha=1}^{\infty} \frac{M\left(p^{\alpha}\right)}{p^{\alpha s}}\right)
$$

by Euler's theorem. But, because

$$
M\left(p^{\alpha}\right)=\sum_{0 \leq m<\alpha} \frac{1}{\phi\left(p^{\alpha-m}\right)}\left\{\psi\left(p^{m}\right)-\psi\left(p^{m+1}\right)\right\}^{2}+\psi^{2}\left(p^{\alpha}\right)
$$

by (24),

$$
\begin{aligned}
1+\sum_{\alpha=1}^{\infty} & \frac{M\left(p^{\alpha}\right)}{p^{\alpha s}} \\
= & 1+\sum_{m=0}^{\infty}\left\{\psi\left(p^{m}\right)-\psi\left(p^{m+1}\right)\right\}^{2} \sum_{\alpha=1}^{\infty} \frac{1}{\phi\left(p^{\alpha}\right) p^{(\alpha+m) s}}+\sum_{\alpha=1}^{\infty} \frac{\psi^{2}\left(p^{\alpha}\right)}{p^{\alpha s}} \\
= & 1+\left(1-\frac{1}{p}\right)^{-1} \sum_{m=0}^{\infty} \frac{\left\{\psi\left(p^{m}\right)-\psi\left(p^{m+1}\right)\right\}^{2}}{p^{m s}} \sum_{\alpha=1}^{\infty} \frac{1}{p^{\alpha(s+1)}}+\sum_{\alpha=1}^{\infty} \frac{\psi^{2}\left(p^{\alpha}\right)}{p^{\alpha s}} \\
= & 1+\left(1-\frac{1}{p}\right)^{-1} \frac{1}{p^{s+1}}\left(1-\frac{1}{p^{s+1}}\right)^{-1} \sum_{m=0}^{\infty} \frac{\left\{\psi\left(p^{m}\right)-\psi\left(p^{m+1}\right)\right\}^{2}}{p^{m s}} \\
& +\sum_{m=1}^{\infty} \frac{\psi^{2}\left(p^{m}\right)}{p^{m s}}
\end{aligned}
$$

whence

$$
\left(1-\frac{1}{p^{s+1}}\right)\left(1+\sum_{\alpha=1}^{\infty} \frac{M\left(p^{\alpha}\right)}{p^{\alpha s}}\right)
$$




$$
\begin{aligned}
= & 1-\frac{1}{p^{s+1}}+\left(1-\frac{1}{p}\right)^{-1} \frac{1}{p^{s+1}} \sum_{m=0}^{\infty} \frac{\left\{\psi\left(p^{m}\right)-\psi\left(p^{m+1}\right)\right\}^{2}}{p^{m s}} \\
& +\sum_{m=1}^{\infty} \frac{\psi^{2}\left(p^{m}\right) p}{p^{m s+1}}-\sum_{m=2}^{\infty} \frac{\psi^{2}\left(p^{m-1}\right)}{p^{m s+1}} \\
= & 1+\left\{-1+\left(1-\frac{1}{p}\right)^{-1}\{1-\psi(p)\}^{2}+\psi^{2}(p) p\right\} \frac{1}{p^{s+1}} \\
& +\sum_{m=2}^{\infty}\left\{\left(1-\frac{1}{p}\right)^{-1}\left\{\psi\left(p^{m-1}\right)-\psi\left(p^{m}\right)\right\}^{2}\right. \\
& \left.+\psi^{2}\left(p^{m}\right) p-\psi^{2}\left(p^{m-1}\right)\right\} \frac{1}{p^{m s+1}} .
\end{aligned}
$$

In this, the coefficient of $p^{-s-1}$ is

$$
\begin{aligned}
\left(1-\frac{1}{p}\right)^{-1}\left\{-1+\frac{1}{p}+1-2 \psi(p)+\right. & \left.\psi^{2}(p)+(p-1) \psi^{2}(p)\right\} \\
& =\frac{1}{p-1}\{p \psi(p)-1\}^{2}=\frac{\eta_{p}^{2}}{p-1}
\end{aligned}
$$

in the notation of (21), while, similarly, that of $p^{-m s-1}$ for $m \geq 2$ is

$$
\frac{1}{p-1}\left\{p \psi\left(p^{m}\right)-\psi\left(p^{m-1}\right)\right\}^{2}=O\left(\frac{1}{p^{2 m-1}}\right)
$$

by (16). Therefore, for $\sigma>0$ in the first instance,

$$
\begin{aligned}
F(s) & =\zeta(s+1) \prod_{p}\left(1+\frac{1}{p-1} \sum_{m=1}^{\infty} \frac{\left\{p \psi\left(p^{m}\right)-\psi\left(p^{m-1}\right)\right\}^{2}}{p^{m s+1}}\right) \\
& =\zeta(s+1) \Phi(s), \quad \text { say },
\end{aligned}
$$

in which the product defining $\Phi(s)$ is absolutely convergent for $\sigma \geq-1$ in virtue of (25), (22), and (26). Moreover, $\Phi(s)$ is regular for $\sigma>-1$ and is represented by a Dirichlet's series

$$
\sum_{n=1}^{\infty} \frac{a_{n}}{n^{s}}
$$

with non-negative coefficients that is absolutely convergent for $\sigma \geq-1$, the right-hand side of (27) providing the analytic continuation of $F(s)$ into the half-plane $\sigma>-1$.

The final ingredient in the treatment of $T^{*}(u)$ is the following lemma, which is probably familiar and which is easily established by partial summation. 
LEMmA. If the series $\sum_{n=1}^{\infty} b_{n}$ with non-negative coefficients be convergent, then, as $u \rightarrow \infty$,

$$
\sum_{n \leq u} b_{n} n^{1 / 2-i t}=o\left(u^{1 / 2}\right) \quad \text { and } \quad \sum_{n>u} \frac{b_{n}}{n^{1 / 2+i t}}=o\left(u^{-1 / 2}\right)
$$

uniformly with respect to $t$.

Being ready to evaluate $T^{*}(u)$ for $u \geq 1$, we first deduce from (17) and (27) that

$$
T^{*}(u)=\frac{1}{2 \pi i} \int_{c-i \infty}^{c+i \infty} \zeta(s+1) \Phi(s) \frac{u^{s}}{s(s+1)(s+2)} d s \quad(c>0)
$$

and then use the relation

$$
\zeta(s+1)=O\left(|t|^{1+\varepsilon}\right) \quad(|t|>1 ; \sigma \geq-3 / 2)
$$

to obtain

$$
\begin{aligned}
T^{*}(u) & =R+\frac{1}{2 \pi i} \int_{-1 / 2-i \infty}^{-1 / 2+i \infty} \zeta(s+1) \Phi(s) \frac{u^{s}}{s(s+1)(s+2)} d s \\
& =R+I(u), \quad \text { say, }
\end{aligned}
$$

where $R$ is the residue of the integrand at $s=0$. Secondly, since the principal part of the expansion of $\zeta(s+1) / s$ about $s=0$ is $1 / s^{2}+\gamma / s$,

$$
\begin{aligned}
R & =\left\{\frac{d}{d s}\left(\frac{\Phi(s) u^{s}}{(s+1)(s+2)}\right)+\frac{\gamma \Phi(s) u^{s}}{(s+1)(s+2)}\right\}_{s=0} \\
& =\frac{1}{2} \Phi(0) \log u+\frac{1}{2} \Phi(0)\left(\frac{\Phi^{\prime}(0)}{\Phi(0)}-\frac{3}{2}+\gamma\right) \\
& =\frac{1}{2} \Phi(0) \log u+\frac{1}{2} B, \quad \text { say. }
\end{aligned}
$$

Next we use the lemma and the convergence of (28) at $s=-1$ to express $\Phi(s)$ in the integrand of $I(u)$ as

$$
\sum_{n \leq u} \frac{a_{n}}{n^{s}}+\sum_{n>u} \frac{a_{n}}{n^{s}}=\Phi_{1}(s)+o\left(u^{-1 / 2}\right)
$$

the limit process indicated by the $o$-symbol being still uniform in $t$. Hence

$$
I(u)=\frac{1}{2 \pi i} \int_{-1 / 2-i \infty}^{-1 / 2+i \infty} \zeta(s+1) \Phi_{1}(s) \frac{u^{s}}{s(s+1)(s+2)} d s+o\left(\frac{1}{u}\right),
$$

whence, moving the line of integration to $\sigma=-3 / 2$ with the aid of (29) and 
the equality $\zeta(0)=-1 / 2$, we get

$$
\begin{aligned}
I(u) & =\frac{\Phi_{1}(-1)}{2 u}+\frac{1}{2 \pi i} \int_{-3 / 2-i \infty}^{-3 / 2+i \infty} \zeta(s+1) \sum_{n \leq u} \frac{a_{n}}{n^{s}} \cdot \frac{u^{s}}{s(s+1)(s+2)} d s \\
& =\frac{\Phi_{1}(-1)}{2 u}+o\left(\frac{1}{u}\right)=\frac{\Phi(-1)}{2 u}+o\left(\frac{1}{u}\right)
\end{aligned}
$$

by another application of the lemma and then by the convergence of the series defining $\Phi(-1)$. In summation, we then extract from this, (30), and (31) the conclusion that

$$
T^{*}(u)=\frac{1}{2} \Phi(0) \log u+\frac{1}{2} B+\frac{\Phi(-1)}{2 u}+o\left(\frac{1}{u}\right)
$$

as $u \rightarrow \infty$.

Similarly, we obtain

$$
T(u)=\Phi(0) \log u+B_{1}+O\left(u^{-1 / 3}\right)
$$

by a contour integral method that does not involve the finer properties of $\Phi(s)$ related to $(22)$ and the lemma. Alternatively, since $u^{2} T^{*}(u)$ is the second Cesàro mean of $T(u)$, this result can be derived from a fairly crude form of (32) by the use of a well-known Tauberian argument.

Our theorem is almost to hand. Letting the $o$-symbol relate to the passage of $x / Q_{2}$ to $\infty$, we deduce from (17), (32), (14), and (7) that

$J\left(x ; Q_{1}, Q_{2}\right)=\frac{1}{2} C^{2} \Phi(0) x^{2} \log \frac{Q_{2}}{Q_{1}}-\frac{1}{2} C^{2} \Phi(-1) Q_{2} x+O\left(x^{2} \log ^{-A} x\right)+o\left(Q_{2} x\right)$

and hence from (12) that

$$
\begin{aligned}
\Gamma\left(x ; Q_{1}, Q_{2}\right)= & C^{2} \Phi(0) x^{2} \log \frac{Q_{2}}{Q_{1}}+\left\{C-C^{2} \Phi(-1)\right\} Q_{2} x \\
& +O\left(x^{2} \log ^{-A} x\right)+o\left(Q_{2} x\right) .
\end{aligned}
$$

Therefore, since

$$
T\left(Q_{2}\right)-T\left(Q_{1}\right)=\Phi(0) \log \frac{Q_{2}}{Q_{1}}+O\left(\frac{1}{\log ^{A} x}\right)
$$

by (33) and (7), we conclude from (11) and (8) that

$$
G\left(x, Q_{2}\right)=\left\{C-C^{2} \Phi(-1)\right\} Q_{2} x+O\left(x^{2} \log ^{-A} x\right)+o\left(Q_{2} x\right)
$$

in the case $Q_{2}>x \log ^{-A} x$ that required attention. But, if $Q_{2}=x$, then a more helpful result is derived by using $J\left(x ; Q_{1}, x\right)=J\left(x, Q_{1}\right)$ in place of (14), the minor changes in the ensuing calculations then leading to

$$
G(x, x)=\left(C+C^{2} B\right) x+O\left(x^{2} \log ^{-A} x\right)
$$

when the notation of (31) is used. 
We have thus substantiated our introductory assertions (2) and (3) in the form of the following

THEOREM. For any sequence (of strictly positive density) satisfying Criteria $\mathrm{U}$ and $\mathrm{S}$, we have

$$
\sum_{k \leq Q} \sum_{0<a \leq k}[S(x ; a, k)-x g\{k,(a, k)\}]^{2}=\left(D_{1}+o(1)\right) Q x+O\left(x^{2} \log ^{-A} x\right)
$$

for $Q \leq x$ and any given positive constant $A$, where

$$
D_{1}=C-C^{2} \prod_{p}\left(1+\frac{1}{p-1} \sum_{m=1}^{\infty} p^{m-1}\left\{p \psi\left(p^{m}\right)-\psi\left(p^{m-1}\right)\right\}^{2}\right)
$$

and where $o(1) \rightarrow 0$ as $x / Q \rightarrow \infty$. Also

$$
\sum_{k \leq x} \sum_{0<a \leq k}[S(x ; a, k)-x g\{k,(a, k)\}]^{2}=D_{2} x^{2}+O\left(x^{2} \log ^{-A} x\right),
$$

where

$$
D_{2}=C-C^{2}\left\{3 \Phi(0)-\gamma \Phi(0)-\Phi^{\prime}(0)\right\}
$$

in the notation of $(27)$.

In interpreting this result, we should first mention that, save when $Q=x$, we are not supplied with anything substantially better than our previous (1) in situations where $Q$ does not differ much from $x$. This lacuna, however, can be filled in any typical instance of this scene where $x /(m+1)<Q \leq$ $x / m$ because a finite combination of explicit terms will represent the sum $T^{*}(x / Q)=T^{*}(m)$ that was the source of the unsatisfactory $o(1)$ constituent. The insertion of these terms in the formula will then furnish a satisfactory outcome provided that $C^{2} x^{2} \log x / Q$ be subtracted in compensation.

It is illuminating to refer to some special cases in order to appreciate the significance of the constant $D_{1}$ in the first formula. For instance, if $C=1$, the non-negativity of $D_{1}$ implies that $D_{1}=0$ and

$$
D_{3}=\prod_{p}\left(1+\frac{1}{p-1} \sum_{m=1}^{\infty} p^{m-1}\left\{p \psi\left(p^{m}\right)-\psi\left(p^{m-1}\right)\right\}^{2}\right)=1
$$

so that $\psi\left(p^{m}\right)=1 / p^{m}$ and $\psi(k)=1 / k$ for all $k$, a conclusion that is of course immediate from the given supposition that the sequence $s$ is derived from the sequence of natural numbers by removing a sequence of density zero. On the other hand, $D_{1}$ can be zero even when $C<1$, as is exemplified by the sequence of odd numbers for which $C=1 / 2$ and $\psi(k)=1$ or 0 according as $k$ be odd or even. It being obvious in this case that $S(x ; a, k)-g\{k,(a, k)\} x=$ $O(1)$, the left side of the formula is $O\left(Q^{2}\right)$, which value is consistent with 
the determination of $D_{1}$ as

$$
\frac{1}{2}-\frac{1}{4}\left(1+\frac{1}{2-1}\right)=0 .
$$

A more recondite example is supplied by the sequence of square-free numbers, for which

$$
C=\frac{6}{\pi^{2}}, \quad \psi(p)=\frac{1}{p+1}, \quad \psi\left(p^{\alpha}\right)=0 \quad(\alpha>1),
$$

and therefore

$$
D_{1}=\frac{6}{\pi^{2}}-\frac{36}{\pi^{4}} \prod_{p}\left(1+\frac{1}{p^{2}-1}\right)=\frac{6}{\pi^{2}}-\frac{36}{\pi^{4}} \prod_{p}\left(1-\frac{1}{p^{2}}\right)^{-1}=0 ;
$$

this accords with a theorem due to Croft [1] that states that in this situation

$$
G(x, Q) \sim B_{2} Q^{3 / 2} x^{1 / 2} \quad\left(Q>x^{2 / 3}\right),
$$

a formula of similar type being available by our method through a further study of the corresponding function $F(s)$ in the half-plane to the left of $\sigma>-1$. We should emphasize, however, that the last two sequences are atypical in the context of our theorem even though explicit cases where $D_{1} \neq 0$ are not easy to isolate naturally. We are therefore content to cite the sequence that, for $\frac{1}{2} N(N+1)<s \leq \frac{1}{2}(N+1)(N+2)$, consists of all odd or even integers in that interval according as $N$ be odd or even; here $C=1 / 2$ and $D_{1}=1 / 4$.

A further study of the product $D_{3}$ would not be without interestespecially in regard to the question of when $D_{1}$ vanishes. A useful point of origin might well be an investigation into the size of $p \psi\left(p^{m}\right)-\psi\left(p^{m-1}\right)$ for $m>1$ whose trivial bound $O\left(1 / p^{m-1}\right)$ was used when establishing the convergence of $D_{3}$. Here there is a relevance to the technique of comparison that was introduced to investigate the entity $\eta_{p}$ appearing in (21). For example, let us now contemplate, for a given set $\mathcal{S}$ of primes $p$, the effect of eliminating every multiple of each prime $p$ save those divisible by $p^{2}$, which are to be affected by the multiplier $p$, that is to say, the result of affecting each member $m$ of a sequence by a non-negative multiplicative weight

$$
\sum_{d_{2} \mid m} \varrho_{d_{2}}
$$

where $d_{2}$ is a product of primes in $\mathcal{S}$ and $\varrho_{d_{2}}$ is the multiplicative function defined by

$$
\varrho_{p^{\alpha}}= \begin{cases}-1 & \text { if } \alpha=1 \\ p & \text { if } \alpha=2 \\ 0 & \text { if } \alpha>2\end{cases}
$$


Then, comparing much as before the results of this particular process on the two sequences in question, we get the inequality

$$
\prod_{p \in \mathcal{S}}\left(1-\psi(p)+p \psi^{2}(p)\right) \leq \frac{1}{C} \prod_{p \in \mathcal{S}}\left(1-\frac{1}{p}+\frac{1}{p}\right)=\frac{1}{C}
$$

that is analogous to (23) and that forms an important part in the proof that

$$
\sum_{p}\left|p \psi^{2}(p)-\psi(p)\right|
$$

is convergent.

\section{References}

[1] M. J. Croft, Square-free numbers in arithmetic progressions, Proc. London Math. Soc. (3) 30 (1975), 143-159.

[2] C. Hooley, On the Barban-Davenport-Halberstam Theorem: I, J. Reine Angew. Math. 274/275 (1975), 206-223.

[3] -, On the Barban-Davenport-Halberstam Theorem: III, J. London Math. Soc. (2) 10 (1975), 249-256.

[4] -, On a new approach to various problems of Waring's type, in: Recent Progress in Analytic Number Theory, Vol. 1, Academic Press, 1981, 127-192.

School of Mathematics

University of Wales, Cardiff

Senghennydd Road

Cardiff CF2 4YH

S. Wales, U.K. 